\title{
VIVENCIANDO SITUAÇÕES DE CONFLITO NO CONTEXTO DA ENFERMAGEM: O ESQUETE COMO ESTRATÉGIA DE ENSINO- APRENDIZAGEM
}

\author{
Experiencing conflict situations in the context of nursing: the use of sketch as a \\ teaching-learning strategy \\ Experiencias con situaciones de conflicto en el contexto de la enfermería: el sketch \\ como estrategia de enseñanza-aprendizaje
}

\author{
Carla Aparecida Spagnol \\ Luciana Alves Silveira Monteiro² \\ Joana Melillo Bastos ${ }^{4}$ \\ Joana Angélica Grossi Honorato ${ }^{5}$
}

Camila Leopoldino de Paula ${ }^{3}$

\section{RESUMO}

Na disciplina optativa: "Análise de situações de conflito vivenciadas pela equipe de enfermagem no contexto das organizações de saúde", do Curso de Graduação da Escola de Enfermagem da Universidade Federal de Minas Gerais, utilizou-se o esquete como técnica de ensino, a fim de possibilitar uma relação pedagógica prazerosa, a partir de vivências apreendidas na realidade. 0 objetivo foi analisar a experiência dos alunos que cursaram a disciplina e dramatizaram uma situação real de conflito. Para isso, um grupo entrevistou um enfermeiro de um serviço de saúde, buscando apreender um conflito vivenciado no seu ambiente de trabalho, a fim de dramatizar essa situação. Um outro grupo, após análise, deveria elaborar outro desfecho para a situação. As encenações em relação à temática gestão de conflitos organizacionais permitiram aos alunos colocarem-se na posição do outro e fazerem reflexões mais elaboradas da prática profissional, refletindo sobre suas condutas como futuros enfermeiros.

Palavras-chave: Educação em Enfermagem. Formação de recursos humanos. Conflito (Psicologia).

\begin{abstract}
In order to provide a pleasurable pedagogical relationship, from experiences perceived in reality, sketches were used as a teaching technique in the elective course "Analysis of conflict situations experienced by nursing staff in the context of health care organizations", from the Undergraduate Nursing Program of the School of Nursing of the Federal University of Minas Gerais. It aimed to evaluate the experiences of students enrolled in the course and who dramatized a real conflict situation. One group interviewed a practice nurse in a health service, seeking to understand a conflict experienced in their work environment, in order to dramatize the situation as it happened. Another group, after analysis, should produce another outcome to the situation. The sketches related to the issue of organizational conflict management enabled students to put themselves in others' position and to have more elaborated reflections of the professional practice, reflecting on their conducts as future nurses.
\end{abstract}

Keywords: Education, Nursing. Human Resources Formation.Conflict (Psychology).

\section{Resumen}

El sketchfue utilizado como técnica de enseñanza en el curso electivo "Análisis de situaciones de conflictos experimentadas por el equipo de enfermería en el contexto de las organizaciones de salud", del Curso de Graduación en Enfermería de la Escuela de Enfermería de la Universidade Federal de Minas Gerais, para proporcionar una relación pedagógica productiva a partir de experiencias extraídas de la realidad. El estudio analizó las experiencias de estudiantes que completaron la disciplina y dramatizaron una situación real de conflicto. Uno de los grupos entrevistó un enfermero de un servicio de salud, buscando entender un conflicto experimentado en su entorno de trabajo, con el fin de dramatizar la situación tal como sucedió. Otro grupo, después del análisis, ha tenido que producir otro resultado para la situación. Las simulaciones relacionadas con el tema de la gestión de conflictos organizacionales permitieron a los estudiantes ponerse en la condición del otro y hacer reflexiones más elaboradas de la práctica profesional, reflejando acerca de sus conductas como futuros enfermeros.

Palabras clave: Educación en Enfermería. Formación de Recursos Humanos. Conflicto (Psicología)

\footnotetext{
${ }^{1}$ Professora Adjunta das disciplinas de Administração em Enfermagem da Escola de Enfermagem da Universidade Federal de Minas Gerais. Doutora em Saúde Coletiva. Belo Horizonte-MG. Brasil. Email: spagnol@ufmg.br; ${ }^{2}$ Enfermeira, Bacharel e Licenciada pela Escola de Enfermagem da Universidade Federal de Minas Gerais. Belo Horizonte-MG. Brasil.. Email: lualves.ufmg@gmail.com; ${ }^{3}$ Acadêmica da Escola de Enfermagem da Universidade Federal de Minas Gerais. Bolsista do Programa Especial de Graduação- PEG da Pró-Reitoria de Graduação da Universidade Federal de Minas Gerais-UFMG. Belo HorizonteMG. Brasil. Email: mimiladepaula@hotmail.com; ${ }^{4}$ Acadêmica da Escola de Enfermagem da Universidade Federal de Minas Gerais. Bolsista de iniciação científica- FAPEMIG. Belo Horizonte-MG. Brasil. Email: joanamelillo@hotmail.com $;^{5}$ Acadêmica da Escola de Enfermagem da Universidade Federal de Minas Gerais. Bolsista do Programa Especial de Graduação- PEG da Pró-Reitoria de Graduação da Universidade Federal de Minas Gerais-UFMG. Belo HorizonteMG. Brasil. Email: joana_honorato@yahoo.com.br
} 


\section{INTRODUÇÃO}

0 foco desse estudo são os conflitos organizacionais, fenômeno que interfere na dinâmica do processo de trabalho e das relações interpessoais se não for analisado e gerenciado de forma adequada. Causa diversos problemas no clima organizacional, nos processos de mudança, na comunicação, na agilidade do processo decisório, na produtividade dos trabalhadores, enfim, no atendimento prestado aos usuários ${ }^{1}$.

Um estudo realizado com gerentes brasileiros evidenciou que o item "lidar com conflito" foi um dos três aspectos mais fracos da competência interpessoal desses profissionais, tanto em relação à sua autopercepção quanto à heteropercepção de seus superiores e subordinados. Esse resultado demonstra a necessidade de se desenvolver a habilidade de administrar e resolver conflitos, como um dos pontos fundamentais da competência interpessoal, o que sugere um maior investimento nessa área de formação profissional?

Para gerenciar a assistência prestada, o enfermeiro deve ser um profissional que precisa se capacitar e se qualificar para desenvolver determinadas competências e habilidades para liderar a equipe de técnicos e auxiliares, ou seja, desenvolver sua capacidade de comunicação, escuta, observação, negociação, senso crítico e sensibilidade para analisar o "outro" e o contexto em que está inserido.

No que diz respeito ao preparo e à capacitação dos enfermeiros para lidarem com as situações de conflito que ocorrem nos diversos serviços de saúde, a literatura evidencia que esses profissionais não são e não estão preparados para gerenciar os conflitos vivenciados no ambiente de trabalho. Frequentemente ignoram a existência desse fenômeno na equipe de enfermagem ou, então, utilizam estratégias apenas para amenizar a situação, ou, ainda, punir os trabalhadores ${ }^{3}$.

0 tema conflito organizacional faz parte do programa da disciplina Administração em Enfermagem II, ministrada aos alunos do $7^{\circ}$ período do Curso de Graduação em Enfermagem da Escola de Enfermagem da Universidade Federal de Minas Gerais (EE-UFMG). Esse tema é abordado em quatro horas; até 0 ano de 2000, utilizava-se a aula expositiva como estratégia de ensino, com o auxílio de slides, apresentando os seguintes aspectos: conceitos de conflito, fontes prováveis de conflito, categorias de conflito existentes e estratégias utilizadas pelo gerente para lidar com situações dessa natureza.

As avaliações dos alunos, em relação às aulas ministradas acerca da referida temática, mostraram que eles não conseguiam fazer a articulação do tema com a prática profissional do enfermeiro, visto que durante as aulas elaboravam diversas questões, principalmente sobre como esse profissional poderia, no cotidiano de trabalho, utilizar determinadas estratégias de resolução de conflito, descritas na teoria. Por diversas vezes, o aluno passava a impressão de que esperava do professor uma receita pré-elaborada ao final da aula.
Entende-se que ensinar aos alunos como o enfermeiro deve mediar conflitos não é tarefa fácil, pois trata-se de questões que dependem da situação em foco, das pessoas envolvidas e do contexto em que ocorreu. Enfim, são vários os fatores que interferem nesse processo complexo e dinâmico das relações interpessoais.

No entanto, o que se observa em relação à educação gerencial na enfermagem, e especificamente à temática conflito organizacional, é que a teoria ministrada em sala de aula, muitas vezes, está distante da prática profissional realizada nas organizações de saúde. Neste sentido, a escola acaba privilegiando o ensino teórico em detrimento do prático, o que afasta os alunos da realidade vivenciada pela equipe de enfermagem nos serviços de saúde, resultando no despreparo e na insegurança do enfermeiro para lidar, principalmente, com o conflito no contexto organizacional. Além disso, algumas estratégias de ensino-aprendizagem não são motivadoras e não levam os alunos a problematizarem a prática profissional ${ }^{4}$.

Sendo assim, o docente na área da enfermagem deve criar situações de aprendizagem que propicie "experiências intensas e adequadas, capazes de despertar no aluno a motivação para a pergunta, para uma atitude investigativa que busque soluções e que fundamente sua intervenção na realidade"5:12. Enfim, o professor deve estimular a capacidade crítico-criativa dos estudantes e promover a liberdade, desafiando a razão ${ }^{5}$.

A capacitação docente, desse modo, deve favorecer uma revitalização constante do processo de ensino-aprendizagem, buscando-se estratégias metodológicas de ensino que transformem a prática educativa e de fato possibilite que os alunos da graduação desenvolvam competências e habilidades para atuarem no mercado de trabalho de forma crítica e reflexiva.

Em virtude da complexidade do conteúdo gestão de conflitos no contexto organizacional, do tempo exíguo estipulado para se trabalhar essa temática no Curso de Graduação em Enfermagem da EE-UFMG e da necessidade de se utilizarem estratégias de ensino dinâmicas e que sejam baseadas em situações reais, sintonizadas com a realidade dos serviços de saúde, foi elaborada a disciplina optativa: Análise de situações de conflito vivenciadas pela equipe de enfermagem no contexto das organizações de saúde.

No cenário da referida disciplina, a utilização de princípios do modelo de Educação de Laboratório mostrouse adequada para auxiliar os alunos na busca de soluções para a complexidade do campo relacional do trabalho em saúde, de modo a assegurar a capacitação pessoal de forma adequada e conciliar a teoria à prática, buscando mudanças de valores, de comportamentos e no exercício das atividades, além de possibilitar uma relação pedagógica diferenciada e prazerosa, a par tir de vivências concretas apreendidas na realidade.

A Educação de Laboratório é um termo genérico, aplicado a um conjunto de metodologias de ensino que buscam 
"mudanças pessoais a partir de aprendizagens baseadas em experiências diretas ou vivências":5:

A metodologia de ensino supracitada visa, por meio de trabalhos e tarefas a serem desenvolvidas individualmente e/ ou em grupo, trabalhar com situaç̃es bem próximas à realidade do mercado profissional, objetivando um maior equilíbrio emocional, a partir da vivência de situações que podem vir a ocorrer no ambiente organizacional. Parte do princípio de que, vivenciando situações, é possível conhecer melhor limites, preferências e características pessoais, possibilitando ao futuro profissional atuar de forma diferenciada ${ }^{6}$.

0 processo vivencial de aprendizagem na metodologia de Educação de Laboratório abrange quatro etapas: atividade, análise, conceituação e conexão com o real (CAVE). A etapa da atividade é realizada por meio de vivências de uma situação significativa para os alunos que pode ser a partir da resolução de um problema, jogos dramáticos, simulações, jogos de papéis, exercícios verbais ou não verbais ${ }^{7}$.

0 relato da experiência da Oficina de Sensibilização para o Desenvolvimento de Habilidades Gerenciais, realizada no modelo de Educação de Laboratório com os enfermeiros gerentes de um hospital-escola de Goiás, evidenciou que a utilização de exercícios vivenciais estruturados, exposição dialogada, filmes, estudos de texto e jogos dramáticos, foram técnicas de ensino fundamentais para se alcançar os objetivos propostos no plano de capacitação e educação permanente do Serviço de Enfermagem do referido hospital. Assim, essa experiência mostrou o uso da Educação de Laboratório como uma estratégia que proporciona os profissionais vivenciarem seus "dilemas, anseios e potencialidades, em um clima de segurança e confiança, propício para a experimentação de mudança e de reflexões sobre o modo de funcionamento das pessoas e dos grupos" $8: 582$.

Tendo como referência os estudos e relatos de experiência encontrados na literatura, acerca da metodologia da Educação de Laboratório, optou-se por utilizar a técnica teatral do esquete, na disciplina "Análise de situações de conflito vivenciadas pela equipe de enfermagem no contexto das organizações de saúde".

0 esquete é uma técnica teatral caracterizada, sobretudo, por uma apresentação ligeira capaz de promover entendimento e suscitar humor, a partir da sátira, às vezes literária, às vezes grotesca, da vida contemporânea ou de uma realidade ${ }^{9}$. Essa técnica foi utilizada como uma das estratégias de ensino da referida disciplina optativa devido a sua viabilidade em termos de montagem e duração, além de ser considerada uma cena curta que apresenta uma situação geralmente cômica, interpretada por um pequeno número de atores sem caracterização aprofundada.
0 presente artigo tem como objetivo analisar a experiência dos alunos que cursaram a referida disciplina optativa e que vivenciaram uma situação real de conflito por meio da utilização de uma técnica teatral - o esquete.

\section{O ESQUETE COMO ESTRATÉGIA DE ENSINO- APRENDIZAGEM: ANÁLISE DE ROTEIROS E CENAS}

Para o ensino de administração em que aspectos práticos e vivenciais são tão importantes quanto o embasamento teórico, o jogo teatral pode ser extremamente vantajos $0^{10}$, pois, com a criatividade e a dramatização, os estudantes podem viver a realidade de forma transfigurada, utilizando as técnicas cênicas para a integração à realidade. As encenações ressaltam as diferentes interpretações de um mesmo gesto, um olhar ou uma atitude, conscientizando sobre a importância da relação com o outro e da compreensão dos diferentes pontos de vista existentes na gestão ${ }^{11}$.

Assim, nota-se que a dramatização no ensino de administração evidencia que as respostas advindas do jogo cênico são mais próximas da realidade, pois a elaboração de cenas é uma maneira de rejeitar a busca de uma solução única e perfeita, ou seja, ao brincar com uma situação real e transcender os fatos do livro didático, os estudantes se expõem ao erro, assumem riscos, negociam entre eles mesmos e com os professores uma solução vivida e sentida ${ }^{10}$.

Para a elaboração do esquete, os alunos matriculados na disciplina optativa foram divididos em dois grupos de seis componentes cada, sendo que o primeiro grupo entrevistou um enfermeiro de um hospital universitário de grande porte da cidade de Belo Horizonte-MG, buscando apreender uma situação de conflito vivenciada por esse profissional.

A entrevista com o enfermeiro foi transcrita na íntegra, e, a partir do seu conteúdo, os alunos elaboraram o roteiro de um esquete, tendo em vista a reprodução fiel da situação de conflito descrita. Essa encenação foi filmada para ser utilizada pelo segundo grupo que, de posse da gravação, foi incumbido de analisar a estratégia de resolução do conflito dramatizada pelo primeiro grupo e elaborar outro desfecho, ou seja, os alunos deveriam buscar outras estratégias para lidar com a situação em foco.

Ao final da disciplina foi aplicado um instrumento avaliativo, no qual os acadêmicos matriculados responderam cinco questões abertas sobre o conteúdo, a estratégia metodológica e a técnica de ensino utilizados no processo de ensino-aprendizagem, além de investigar se a metodologia da Educação de Laboratório e o esquete contribuíram para a articulação e aproximação da teoria sobre conflito e a prática profissional.

Ressalta-se que essa disciplina está vinculada ao projeto de pesquisa intitulado "Vídeo educativo sobre situações de conflito vivenciadas pela equipe de enfermagem no contexto da 
instituição de saúde", que foi aprovado pelo Comitê de Ética em Pesquisas da Universidade Federal de Minas Gerais, sob o número de protocolo: 473/07. Sendo assim, tanto o enfermeiro que concedeu a entrevista quanto os alunos que responderam ao instrumento avaliativo da disciplina assinaram o Termo de Consentimento Livre e Esclarecido, garantindo o sigilo e o anonimato dos participantes.

Para a elaboração do roteiro do esquete, os alunos do grupo 01 fizeram uma adaptação da situação narrada pela enfermeira entrevistada, apresentando pequenas modificações. Na situação real que foi dramatizada, a enfermeira utilizou a estratégia de supressão e um instrumento administrativo - 0 memorando - para lidar com o conflito vivenciado que estava relacionado ao remanejamento de um técnico de enfermagem para outro setor e o seu descumprimento à essa ordem dada pelo enfermeiro, ocasionando a sua demissão.

No esquete apresentado pelo grupo 01 , os alunos tiveram, então, a oportunidade de vivenciar de forma simulada uma situação extrema entre a enfermeira e o técnico de enfermagem, ao estabelecerem uma relação conflituosa entre eles, causada por uma ordem que não foi cumprida. Puderam, ainda, experimentar uma gerência centralizada, em que a enfermeira de plantão repassou seus problemas para a Direção de Enfermagem, ao enviar o memorando descrevendo o fato ocorrido.

A estratégia utilizada pela enfermeira entrevistada foi de supressão, e os alunos, ao dramatizarem essa estratégia, evidenciaram que, nesse caso, o controle do conflito por parte da enfermeira se deu pelo uso da força, sendo caracterizado principalmente pela situação de ganha/perde 2 . Esta forma de lidar com conflito organizacional é fundamentada em uma relação em que uma das partes, sendo mais forte do que a outra, exerce sua autoridade e poder para eliminar o conflito em questão, favorecendo que o mais forte atinja seus objetivos.

As pessoas que estão envolvidas em situações semelhantes ao do caso descrito não se comunicam abertamente e utilizam-se de regras e leis para sempre vencer ${ }^{12}$. A enfermeira protagonista, dramatizada por uma das alunas, por sua vez, atingiu o seu objetivo realizando a demissão do funcionário como uma forma de eliminar o conflito.

A utilização do esquete como estratégia de ensino, além de permitir uma análise mais aprofundada da situação de conflito relatada, possibilitou, ainda, aos alunos analisarem e refletirem sobre o modelo de gestão clássico e como suas características interferem no trabalho da enfermagem, dificultando o processo de comunicação e a tomada de decisão. Nesse caso, houve consequências para o trabalhador, que foi demitido, e para a organização do trabalho, pois houve um desfalque no quadro de pessoal da unidade, trazendo, consequentemente, prejuízos para a assistência de enfermagem.

Ressalta-se que, na realidade, mesmo o hospital sendo público, algumas admissões são feitas por contrato, e não por concurso, o que facilitou o processo de demissão, opção feita pela enfermeira e pela Direção de Enfermagem, em relação ao técnico que desacatou a chefe de plantão.

0 papel da pessoa enquanto chefe condiciona o estilo de lidar com o conflito, pois, muitas vezes, os chefes usam um estilo mais autoritário em um esforço de deterem o controle comportamental dos seus subordinados ${ }^{13}$, o que ocorreu na situação encenada, em que a enfermeira utilizou do seu poder para resolver o conflito com o subordinado, o técnico de enfermagem.

A proposta da disciplina era que os alunos do grupo 02, a partir do roteiro e vídeo produzidos pelo grupo 01, elaborassem outro desfecho para o conflito encenado, pensando em outras possibilidades de resolução dele.

No esquete apresentado pelo grupo 02 , os alunos optaram por dramatizar a estratégia de negociação, reconhecendo o outro e o seu ponto de vista, o que contribui para criar um ambiente de trabalho mais tranquilo, um clima cordial e um entendimento entre as pessoas, em que as diferenças possam conviver de maneira mais pacífica.

A estratégia de negociação é caracterizada pela dupla vitória, ou seja, as partes envolvidas no conflito devem se sentir satisfeitas com o desfecho da resolução. Embora a negociação indique perdas e ganhos para ambas as partes, não existem regras que explicitem que cada uma das partes deva perder ou ganhar a mesma quantia ${ }^{13}$.

Ao utilizar a referida estratégia para a resolução do conflito, os alunos queriam mostrar que "ambas as partes devem sentir que os seus interesses foram tratados com justiça, que os critérios adotados foram utilizados de forma legítima e em condições de igualdade"14:113 para todos.

Os alunos do grupo 02, ao analisarem a situação dramatizada pelo grupo 01, enfatizaram no esquete o diálogo e a reunião como instrumentos gerenciais. Essa escolha corroborou os resultados de um estudo realizado em Unidades Básicas de Saúde do município de Colombo, no Estado do Paraná, em que as autoras mostraram que uma das possibilidades para se reduzir os enfrentamentos dos enfermeiros com a sua equipe e os diferentes níveis hierárquicos seria por meio de reuniões formais e informais que possibilitassem uma comunicação efetiva, propiciando "a formação de vínculos de confiança, tolerância, bom humor, troca de informações, ajuda, apoio e cordialidade", o que contribui para se alcançar certo equilibrio nas relações interpessoais ${ }^{15: 188}$.

Nesse caso, o esquete utilizado como estratégia de ensino possibilitou que os alunos realizassem uma reflexão acerca dos princípios que subsidiam os modelos de gestão mais contemporâneos, em que facilitam a comunicação, a tomada de decisão e evidenciam o trabalhador como sujeito do processo de trabalho. Ressaltam a visão de um trabalhador que procura ter controle sobre suas condutas, conhece seus objetivos e busca enunciar seus projetos e desejos, deixando de agir de forma submissa. 
Após a dramatização dos dois grupos, as discussões em sala de aula evidenciaram que o processo de ensinoaprendizagem em grupos pequenos pode propiciar aos discentes um exercício para a prática profissional em que "eles aprendem a escutar, dialogar e compartilhar experiências, além de mudanças no seu modo de ser/atuar para realizar um trabalho em grupo/equipe criativo e crítico"16:54.

Sendo assim, a Educação de Laboratório com a utilização do esquete como técnica de ensino pode ser considerada um modelo de aprendizado teórico-vivencial ${ }^{7}$ que proporcionou a experiência dos grupos com o mundo real da enfermagem, ampliando a sua visão quanto às problemáticas enfrentadas pelo enfermeiro.

Os docentes de enfermagem de uma faculdade privada do interior de São Paulo também utilizaram o teatro como estratégia pedagógica para ministrar alguns conteúdos da disciplina Enfermagem em Saúde Coletiva. Os grupos de alunos, com o auxílio de um professor de teatro, produziram as peças: "A revolta da vacina e a Louca enfermaria", partindo do princípio que essa técnica de ensino permite superar as tradicionais fronteiras estabelecidas entre as disciplinas na busca para a formação dos alunos enquanto cidadãos e sujeitos da história ${ }^{17}$.

No referido estudo, ao avaliarem o significado do teatro para a formação profissional, os discentes reconheceram que essa atividade ar tística permitiu o autoconhecimento, e que se tornaram mais confiantes e autônomos depois que a iniciaram. Por outro lado, ficaram mais flexíveis e menos rígidos nas suas percepções, aceitaram mais abertamente os outros, buscando compreender os seus sentimentos ao representarem papéis que se aproximavam das suas realidades, como se fosse o seu próprio mundo 17:167

Essas possibilidades de aprendizagem a partir das expressões da arte foram relatadas ainda em um estudo que analisou a experiência do ensino da gestão do Sistema Único de Saúde utilizando-se a obra "As Intermitências da Morte", de José Saramago. Segundo as autoras, esse diálogo da arte com a educação mostrou que a "interface entre a produção acadêmica, a teorização das práticas, a criatividade, a abstração ética e estética produz sentidos de aprendizagem que ultrapassam a racionalidade instrumental", contribuindo para a formação dos profissionais de saúde ${ }^{18}$.

Nesta perspectiva, ao ministrar a disciplina optativa "Análise de situações de conflito vivenciadas pela equipe de enfermagem no contexto das organizações de saúde", buscou-se consolidar um processo de ensino-aprendizagem pautado no desenvolvimento da autonomia, do potencial criativo e da capacidade de abstração dos alunos de enfermagem, por meio da interface entre a arte e a educação, possibilitando aprender por meio de experiências vivenciadas a partir de situações reais, e não apenas por meio de teorias, fórmulas e modelos pré-estabelecidos.

\section{CONSIDERAÇÕES FINAIS}

Ao final da disciplina optativa realizou-se uma avaliação sobre a utilização do esquete como estratégia metodológica de ensino e verificou-se que essa técnica possibilitou a articulação de conceitos acerca da temática em foco, com a realidade dos serviços de saúde, pois os conflitos dramatizados foram analisados à luz do referencial teórico e das discussões realizadas em sala de aula.

Segundo os graduandos, a encenação do conflito proporcionou maior aproximação da vida acadêmica/ profissional em relação à temática gestão de conflitos organizacionais, pois permitiu se colocarem na posição do outro e fazerem reflexões mais elaboradas a respeito do assunto vivenciado.

Para os alunos, os pontos facilitadores da elaboração do esquete foram a praticidade e a objetividade dessa técnica teatral, ao passo que os dificultadores foram a timidez de muitos acadêmicos e o encontro dos grupos para o ensaio e a elaboração do roteiro, visto que, por ser uma disciplina optativa, os estudantes são de períodos diferentes.

Os acadêmicos consideraram que a experiência da dramatização do esquete contribuiu para o aprendizado e a sua formação profissional, uma vez que proporcionou 0 desenvolvimento do pensamento crítico sobre o tema e forneceu subsídios para análise mais profunda sobre os tipos e as estratégias de resolução de conflito.

Finalmente, as avaliações dos graduandos mostraram aceitação do esquete como técnica de ensino para o aprendizado, indicando que tal recurso merece ser considerado uma alternativa ao processo de ensinar e de aprender na enfermagem.

\section{REFERÊNCIAS}

1. Falk MLR. A competência gerencial nos conflitos interpessoais. REAd. Rev. Eletronica Adm [on-line]. 2001 Dez; [citado 15 jan2011]; 22 (7): [aprox. 10 telas]. Disponível em: <http://www.read.ea.ufrgs.br/edicoes/ pdf/artigo_118.pdf>.

2. Moscovici F. Desenvolvimento interpessoal: treinamento em grupo. $3^{\mathrm{a}}$ ed. Rio de Janeiro (RJ): Jose Olimpio; 2008.

3. Spagnol CA, Santiago GR, Campos BMO, Badaró MTM, Vieira JS, Silveira APO. Situações de conflito vivenciadas no contexto hospitalar: a visão dos técnicos e auxiliares de Enfermagem. Rev. Esc. Enferm. USP. 2010 set; 44(3): 803-11.

4. Spagnol CA. A trama de conflitos vivenciada pela equipe de enfermagem no contexto da instituição hospitalar: como explicitar seus "nós"? [tese de doutorado]. Campinas (SP): Universidade Estadual de Campinas; 2006. 
5. Costa KNM, Rebouças CBA, Silva GRF, Marrket W. Enfermeiro-docente: aspectos interrelacionados à sua formação. Enferm. glob. 2010 Jun; [citado em 02 de fev 2011]; 19. Disponível em <http://scielo.isciii.es/ scielo.php?pid=S1695-61412010000200017\&script $=$ sci _arttext\&tlng=pt>.

6. Marcheti APC, Ferroni MSN, Oliveira NSS. Educação de laboratório uma estratégia de ensino e aprendizagem alternativa para 0 desenvolvimento técnico e interpessoal do indivíduo. Anais do $29^{\circ}$ Congresso Brasileiro de Ensino de Engenharia; 2001; Rio Grande do Sul (RS), Brasil. Rio Grande do Sul (RS): Faculdade de Engenharia da Pontifícia Universidade Católica do Rio Grande do Sul; 2001; [citado 2011 fev 15]. Disponível em: www.pp.ufu.br/Cobenge2001/trabalhos/MTE128.pdf. [aprox. 7 telas]

7. Munari DB, Merjane TVB, Cruz RMM. Aplicação do modelo de Educação de Laboratório no processo de formação do enfermeiro. Rev. enferm. UERJ. 2005 maio/ago; 13(2): 263-9.

8.Munari DB, Nunes FC, Motta KAMB, Esperidião E, Silva Jl, Coelho MA. Educação de Laboratório como ferramenta no processo de educação continuada de enfermeiros gerentes. Rev. enferm. UERJ. 2008 set/dez; 16(4): 577-83.

9.Eiras NB, Ayres JR, Silva MM, Santos LB, Paulino LF. Esquetes Teatrais: Questões Intergeracionais em Debate. Anais do $2^{\circ}$ Congresso Brasileiro de Extensão Universitária; 2004 set 12-15; Belo Horizonte (MG), Brasil. Belo Horizonte (MG), Brasil: UFMG; 2004 [citado 2011 fev 15]. Disponível em <http://www.ufmg.br/congrext/Cultura/Cultura2.pdf > . [aprox.7 telas]

10. Carvalho JLFS. Estimulando a criatividade e o pensamento crítico: 0 professor como ator, diretor e dramaturgo. In: Davel E, VegaraSC, Ghadiri DP. Administração com arte- experiências vividas de ensino aprendizagem. São Paulo(SP): Ed. Atlas; 2007. p. 173-84.

11. Gosse SCC. 0 uso de técnicas teatrais para desenvolver o saber relacional dos gestores. In: Davel E, Vegara SC, Ghadiri DP. Administração com arte- experiências vividas de ensino aprendizagem. São Paulo: Ed. Atlas; 2007. p. 163-72.

13. McIntyre SE. Como as pessoas gerem o conflito nas organizações: estratégias individuais de negociações. Rev. Análise Psicológica. 2007; 2(25): 295-305.

14. Marquis BL, Huston CJ. Administração e liderança em enfermagem: teoria e aplicação. Regina Machado Garcez e Eduardo Schaan, tradutora. $2^{\mathrm{a}}$ ed. Porto Alegre(RS): Artes Médicas do Sul; 1999.

15. Sales AAR, Lima FRF, Farias FSAB. Refletindo sobre a administração e negociação de conflitos nas equipes de saúde. Rev. bras. promoç. saúde. 2007; 20(2): 111-5.

16. Corradi EM, Zgoda LTRW, Paul MFBP. 0 gerenciamento de conflitos entre a equipe de enfermagem. Cogitare enferm. 2008 Abr/Jun; 13(2): 184-93

17. Barbato RG, Corrêa AK, Souza MCBM. Aprender em grupo: experiência de estudantes de enfermagem e implicações para a formação profissional. Esc Anna Nery. 2010 Jan/Mar; 14(1): 48-55.
18. Camargo RAA. A saúde em cena: o teatro na formação do enfermeiro [tese doutorado]. Ribeirão Preto (SP): Universidade São Paulo; 2006.

19. Pires MRGM, Spagnol CA, Brito MJM, Gazzinelli MF, Montenegro LC. Diálogos entre a arte e a educação: uma experiência no ensino de administração em saúde. Texto \& contexto enferm. 2009; 18(3): 559-67. 